\title{
A FLEXIBLE PH SENSOR ARRAY ON PAPER USING LASER PATTERN DEFINITION AND SELF-ALIGNED LAMINATED ENCAPSULATION
}

\author{
Rahim Rahimi ${ }^{l}$, Manuel Ochoa ${ }^{l}$, Tejasvi Parupudi ${ }^{l}$, Xin Zhao ${ }^{2}$, \\ Mehmet R. Dokmeci ${ }^{2}$, Ali Khademhosseini ${ }^{2}$, and Babak Ziaie ${ }^{1}$ \\ ${ }^{1}$ Purdue University, West Lafayette, Indiana, USA \\ ${ }^{2}$ Harvard-MIT Health Sciences and Technology, Cambridge, MA, USA
}

\begin{abstract}
Chronic wound $\mathrm{pH}$ is a key indicative parameter for assessing the healing progress. Due to their size and inability to sample multiple wound regions independently, commercial $\mathrm{pH}$ meters are not well-suited for spatial maping of wound $\mathrm{pH}$. To address this issue, we present an inexpensive, flexible array of $\mathrm{pH}$ sensors on a paper platform. Each sensor consists of two electrodes screenprinted (using a laser-defined mask) on an acrylic-coated paper (i.e. palette paper), a $\mathrm{Ag} / \mathrm{AgCl}$ reference electrode, and a carbon electrode coated with a conductive proton-selective polymer, (polyaniline, PANI). Laser-machining is further used to create a self-aligned passivation layer that is bonded over the sensors by lamination technology. Characterization of the $\mathrm{pH}$ sensors reveal a linear $\left(\mathrm{r}^{2}=0.9734\right)$ relationship between output potential and $\mathrm{pH}$ for values in the $\mathrm{pH}$ range of $4-10$, with an average sensitivity of $-49.5 \mathrm{mV} / \mathrm{pH}$. The sensors feature a response time of $4 \mathrm{~min}$ and very limited hysteresis over four $\mathrm{pH}$ cycles ( $\mathrm{pH} 4$ to 10 to 4 ).
\end{abstract}

\section{INTRODUCTION}

The $\mathrm{pH}$ level in the wound bed is a key indicative parameter for assessing the healing progress of chronic wounds. Unlike healthy skin or healing acute wounds that have a slightly acidic $\mathrm{pH}$ (5.5-6.5), chronic wounds often exhibit $\mathrm{pH}$ values higher than 7.4 due to alkaline byproducts of bacterial colony proliferation [1]-[3]. Thus, $\mathrm{pH}$ monitoring offers a method for promptly identifying such infections in order to effectively treat them in a timely manner.

Commercial $\mathrm{pH}$ meters can be used in acute or uniformlyhealing wounds, but their practical efficacy in chronic wounds is limited by their inability to sample multiple wound regions independently and efficiently. In many cases, the irregular vascular structure of chronic wounds causes a heterogeneous distribution of infection in the wound bed, resulting in drastic $\mathrm{pH}$ variations throughout the affected area [4]. The design of most commercial $\mathrm{pH}$ meters makes their use impractical for assessing such wounds. Traditionally, these sensors consist of a doped glass membrane (e.g., thin amorphous silicon dioxide with alkali metals). The electric potential across the glass membrane is sensitive to changes in the concentration of $\mathrm{H}^{+}$ions in the solution. The potential difference is measured with respect to a reference electrode that is immersed into the same solution (usually combined with the working electrode into one device) [5]. Although many improvements over the decades have achieved a small device footprint, many drawbacks still remain, including a limitation to single-point measurements, a hard structure that may cause tissue insults, and a lack of mechanical flexibility that prevents it from conforming to the wound bed. A more practical alternative to wound $\mathrm{pH}$ monitoring would be a flexible array of $\mathrm{pH}$ sensors that can cover the wound site and generate a map of $\mathrm{pH}$ levels throughout the wound site, thus revealing the location and concentration of bacterial infections.

To improve compatibility of $\mathrm{pH}$ sensors with soft materials, recent research has produced several sensor designs that further minimize size and increase mechanical flexibility. A common approach the use of ion sensitive field effect transistors (ISFETs)
[6]-[8], which feature a small size and fast response and are suitable for in vivo applications. Their main drawback, however, is the fabrication process which requires time consuming and costly cleanroom processing. An alternate approach is the use of metal oxides (e.g. $\mathrm{IrO}_{\mathrm{x}}, \mathrm{RuO}_{2}, \mathrm{SnO}_{2}$ ) formed on various substrates by processes such as sputtering, electro-deposition, and sol-gel [9][12]. These sensors feature a near-Nernstian performance, potential and thermal stability, and a sensitivity as high as $-77 \mathrm{mV} / \mathrm{pH}$ [13]; however, they usually exhibit significant drift [14].

More recently, researchers have developed polymer-based $\mathrm{pH}$ sensors, including chemo-mechanical sensors based on $\mathrm{pH}$ responsive hydrogels [15]-[17] as well as electrodes coated with a proton-selective polymer film (e.g., polyaniline, polypyrrole) [18], [19]. While the former type boasts unmatched mechanical compliance, its slow response time and requirement for special storage (hydrated) conditions limit its practical utility. The latter type, in contrast, offers a satisfactory degree of flexibility along with a straightforward and inexpensive fabrication process. For these reasons, such conductive polymer-based $\mathrm{pH}$ sensors have shown great promise for biomedical applications.

(a)

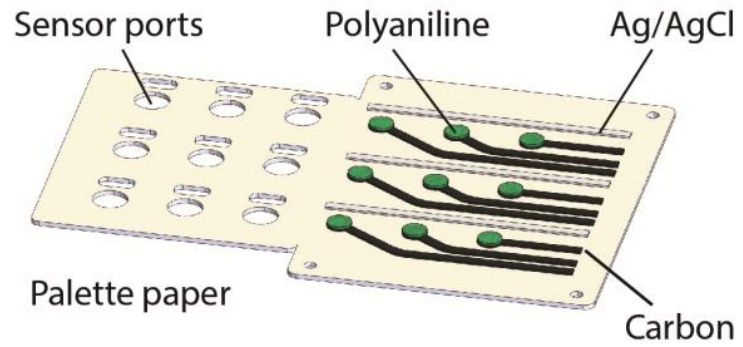

(b)

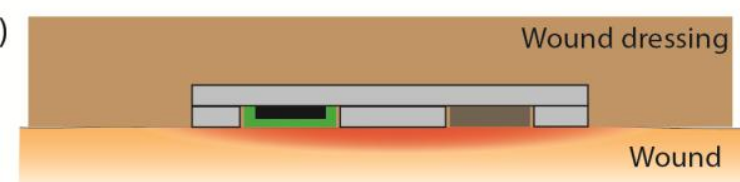

Figure 1: (a) A 3D exploded view of the $3 \times 3 \mathrm{pH}$ sensor array on paper with self-aligned encapsulation. (b) Illustration of its implementation as a component of wound dressings for advanced wound monitoring.

In this work, we followed a similar conductive polymer approach and employed PANI to develop an inexpensive and flexible array of $\mathrm{pH}$ sensors on a paper substrate. The sensor array features fabrication using commercial technologies for rapid prototyping. Figure 1 shows a schematic of a $3 \times 3$ array of sensors on a paper substrate. Each individual sensor consists of two electrodes, one being a $\mathrm{Ag} / \mathrm{AgCl}$ reference electrode and the other a carbon electrode coated with a conductive proton-selective polymer, polyaniline (PANI). The sensors are screen-printed on an acrylic-coated paper (i.e., palette paper) using a laser-patterned tape as a mask. This method features a simple technique for 
directly patterning the mask on the paper substrate. Laser machining is further used to create a self-aligned passivation layer that is bonded over the sensors by using a commercial laminating machine.

\section{SENSOR DESIGN AND MEASUREMENT}

\section{Theory of operation}

The operation of the $\mathrm{pH}$ sensors is based on the protonation and de-protonation of nitrogen atoms in the polymer chains of PANI [20]. In acidic solutions the polymer is doped with $\mathrm{H}^{+}$ions to create the emeraldine salt (ES) form of PANI, which is known for its high electrical conductivity. This resulting surface charge increases the electrical potential of the electrode relative to the reference. When the polymer is subsequently immersed in an alkaline solution, the captured $\mathrm{H}^{+}$ions are neutralized, resulting in decreased surface charge (and hence potential) on the polymer. The deprotonated or neutralized form of PANI is referred to as its emeraldine base (EB) form and is not electrically conductive. This $\mathrm{pH}$-dependent electrochemical equilibrium between the ES and EB moieties of PANI results in an inverse relationship between the electrochemical potential of the sensing electrode and the $\mathrm{pH}$ of its environment.

\section{Sensor fabrication}

Figure 2 shows the fabrication process of the $\mathrm{pH}$ sensor array. First, a single layer of tape $\left(3 \mathrm{M}^{\circledR}\right.$ MagicTape $\left.{ }^{\mathrm{TM}}\right)$ is attached to a piece of palette paper substrate to act as a mask (Figure $2 b$ ). The electrode mask pattern is then defined on the tape by directly writing on the tape with a laser engraver (Universal Laser Systems, Inc., Scottsdale, AZ). This process ablates the cellulose acetate backing of the tape, leaving behind only the adhesive layer (Figure 2c). To eliminate the need for alignment, the insulating layer is fabricated on the same substrate with the tape mask by laser machining openings on the side of the paper without traces. Next, the carbon and silver patterns are screen-printed onto the palette paper (Figure 2d). The inks are allowed to cure at $100{ }^{\circ} \mathrm{C}$ for $30 \mathrm{~min}$. After curing, the tape mask is peeled off, revealing 9 carbon and 3 silver electrodes on the palette paper.

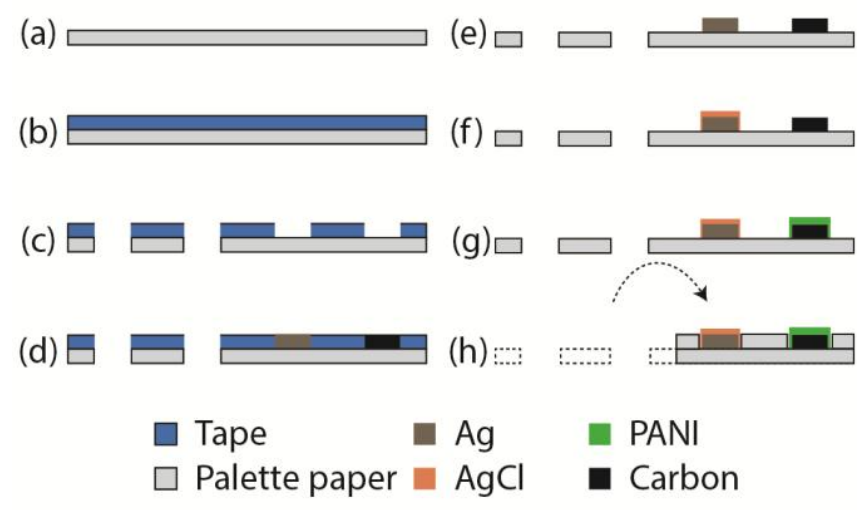

Figure 2: Fabrication process of the sensor: (a-c) laser-pattern tape to act as a direct mask; (d) screen print silver and carbon electrodes; (e) remove tape mask; $(f)$ chloridate silver electrodes as the reference electrodes; (g) drop-cast PANI on carbon electrode with $\mathrm{HCl}$ doping; ( $h$ ) fold the isolation layer over the electrodes, and bond by lamination.

Following that, the silver chloride electrode is prepared by selectively electroplating a layer of silver chloride on the silver electrodes using a current of $1 \mathrm{~mA}$ for $2 \mathrm{~min}$ in a $1.0 \mathrm{M} \mathrm{NaCl}$ solution. After the electrolysis process, the surface of the electrodes is rinsed with de-ionized water and allowed to dry at $70{ }^{\circ} \mathrm{C}$ for at least 3 hours.

Meanwhile, a polyaniline solution is prepared by dissolving $25 \mathrm{mg}$ of polyaniline emeraldine base in $25 \mathrm{~mL}$ dimethyl sulfoxide (DMSO). The base form is used since it is very soluble in organic solvents such as DMSO and ensures uniform coating of the polyaniline on carbon. The polyaniline emeraldine solution is stirred with a magnetic rod for 5 hours before use.

After the electrodes on the paper have dried, a $4 \mu \mathrm{L}$ volume of polyaniline solution (ion selective membrane) is drop-casted on the carbon electrode and allowed to dry on the carbon traces for 24 hours at room temperature. Following that, the PANI is doped with $\mathrm{HCl}$ in a vacuum chamber for 5 hours. The $\mathrm{HCl}$ fumes in the vacuum chamber introduce $\mathrm{H}^{+}$ions into the polyaniline emeraldine base film to produce the polyaniline emeraldine salt [20]. During the doping process the polyaniline base film is protonated changes color from dark blue to dark green, which has a higher conductivity compared to emeraldine base.

The electrodes are then rinsed with de-ionized water and desiccated by heating in an oven at $70{ }^{\circ} \mathrm{C}$ for 3 hours. Finally, the palette paper substrate is folded in half to create a structure with 9 $\mathrm{pH}$ sensor interconnections sandwiched between the two layers of palette paper. Bonding between the two layers of paper is accomplished using a hot roll laminator (Apache AL13P Professional) at $143{ }^{\circ} \mathrm{C}$.

\section{Measurement setup and procedure}

To evaluate the performance of the $\mathrm{pH}$ sensor array, potentiometric measurements were conducted in different $\mathrm{pH}$ buffer solutions from $\mathrm{pH} 4$ to $\mathrm{pH} 10$. For $\mathrm{pH}$ values of 4, 7, and 10, commercial buffer solutions were purchased (Pinnacle pH Buffers, Nova Analytics) and used directly. To achieve a complete range of

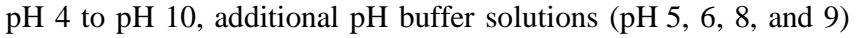
were prepared by mixing different ratios of the commercial solutions. The $\mathrm{pH}$ for all buffer solutions was verified with a commercial $\mathrm{pH}$ meter (Model IQ125, IQ Scientific Instruments, USA) prior to all experiments.

The potentiometric measurements were conducted at room temperature using an Agilent 34401A digital multimeter. The multimeter was set to high impedance mode in order to reduce the current driven from the sensors. A computer was connected to the multimeter via a GPIB interface, and continuous data acquisition was implemented using Agilent's IntuiLink software in combination with Microsoft Excel. The measurements were carried out by immersing the working and reference electrodes of each sensor in different buffer solutions and measuring the potential across the electrodes.

To assess the sensor in terms of stability and repeatability the sensors were tested in titrated cycles from $\mathrm{pH} 4$ to $\mathrm{pH} 10$ and back to $\mathrm{pH}$ 4. The sensors were sequentially placed in different levels of $\mathrm{pH}$ solution (without rinsing the sensors in between solutions). Meanwhile, the potential between the working and reference electrodes was recorded as a function of time at a sampling frequency of $1 \mathrm{~Hz}$.

The conductive traces of individual sensors on the array must remain electrically isolated from each other in a moist wound environment in order to minimize noise in the output of the sensors. To evaluate the reliability of the packaging used here, we investigated current leakage in a modified array of metallic traces on various substrates. The experimental setup for these tests is illustrated in Figure 3. Each test sample consisted of two conductive traces patterned on palette paper and sealed with a thermally laminated layer of various insulating materials $(75 \mu \mathrm{m}-$ thick Kapton ${ }^{\circledR}$ tape, Scotch ${ }^{\circledR}$ MagicTape $^{\mathrm{TM}}$, and palette paper). The 
samples were placed in a phosphate-buffered saline (PBS) solution, to mimic wound fluid. A 1 volt bias was then applied to the two ends of the electrodes using a Hewlett-Packard E3630A DC power supply. Meanwhile, the leakage current between the two electrodes was monitored over a period of 24 hours using a multimeter with the data acquisition setup described above. As control samples, two conductive traces without insulating layer were also tested under the same conditions. In all cases, the solution was stirred constantly during the experiments using a magnetic stirrer.

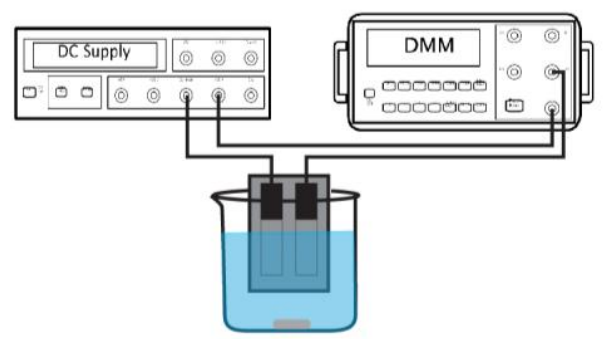

Figure 3: Scheme of the test setup for testing leakage current.

\section{RESULTS AND DISCUSSION}

Figures 4(a-b) show photographs of the sensor array in its final fabrication steps (folding and lamination); the final device is shown in Figure 4c. The resulting array is flexible and comfortably wearable. The working areas of the $\mathrm{pH}$-sensitive electrodes have a $2 \mathrm{~mm}$ diameter. The number and density of sensors can be further adjusted during the design to accommodate specific wound sizes and geometries. Such sensor platform offers versatile adaptability for either low-production patient-customized fabrication or highvolume roll-to-roll manufacturing. The next two subsections discuss the performance of the sensor array.

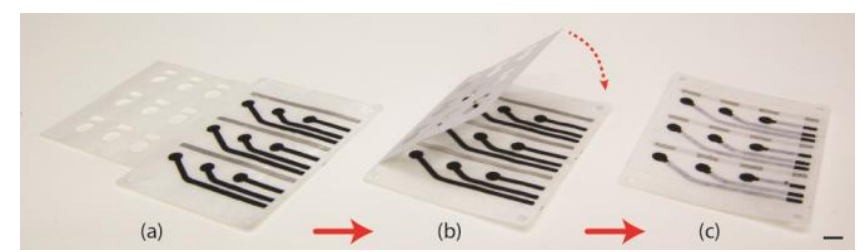

Figure 4: Photograph of the fabricated pH sensor array, (a) fabricated electrodes on paper; $(b)$ folding the encapsulation layer over the electrodes; (c) the final array of $p H$ sensors after lamination. Scale bar: $5 \mathrm{~mm}$.

\section{Sensor performance}

Figure 5 shows the potentiometric response of each sensor as a function of $\mathrm{pH}$. The data reveal a linear $\left(\mathrm{r}^{2}=0.9734\right)$ relationship for 7 different $\mathrm{pH}$ conditions (in the range $\mathrm{pH} 4-10$ ), with average sensitivity of $-49.5 \mathrm{mV} / \mathrm{pH}$. This response is characteristic of Nernstian theory for $\mathrm{pH}$ sensors.

Figure 6 shows the results of the stability and reliability experiments. The sensors exhibit satisfactory stability and produce a distinct response for each $\mathrm{pH}$ condition between $\mathrm{pH} 4$ and $\mathrm{pH} 10$, with very limited hysteresis. Additionally, the average response time of the sensors (to $90 \%$ of the steady-state output potential) is $4 \mathrm{~min}$. The slow response time is attributed to thickness variations of the drop-cast PANI membrane. Although previous reports of PANI-based $\mathrm{pH}$ sensitive electrodes have featured a faster response time (i.e., seconds), their fabrication process employed electro-deposition of PANI [5]. Such techniques are difficult to adapt for arrays of sensors on flexible materials. Drop-casting, in contrast, offers a more favorable approach at the expense of a slower response time. For wound healing applications, however, this tradeoff is justified since the $\mathrm{pH}$ of the wound is not expected to vary drastically over time [1]. Hence, the sensitivity and response time of our sensor render it suitable for integration with in low-cost wound monitoring dressings and microsystems.

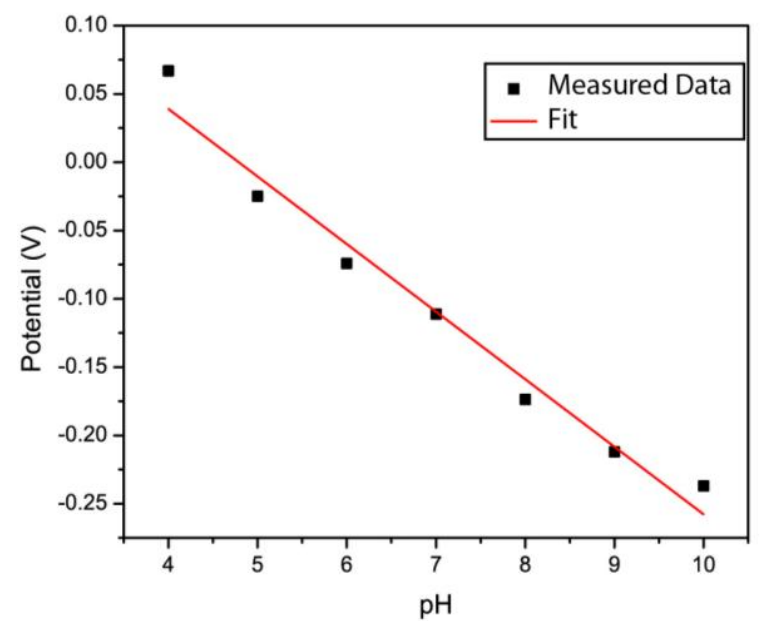

Figure 5: The measured sensor response to various $\mathrm{pH}$ conditions between $\mathrm{pH} 4$ and $\mathrm{pH} 10$. The data show a linear response $\left(r^{2}=0.9734\right)$ with average sensitivity of $-49.5 \mathrm{mV} / \mathrm{pH}$.

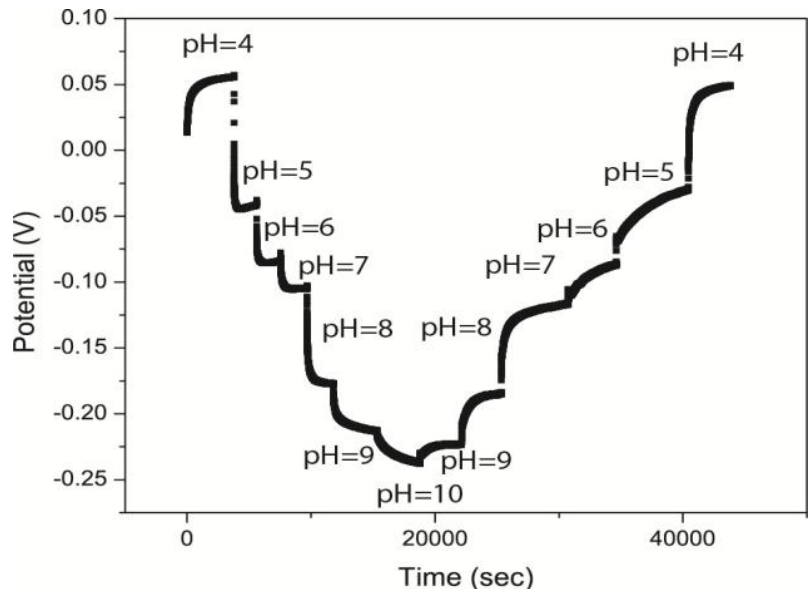

Figure 6: Stability and repeatability of the $\mathrm{pH}$ sensor from $\mathrm{pH} 4$ to 10 and back to 4 . The test was repeated for four cycles. The data show a distinct response for each $\mathrm{pH}$ value, very little drift, and negligible hysteresis.

\section{Leakage current characterization}

The results of the packaging investigations are depicted in Figure 7. The data show that the samples laminated with Kapton ${ }^{\circledR}$ tape and Scotch ${ }^{\circledR}$ tape both exhibited a leakage current after a few hours in PBS (45min for samples with Scotch tape; 13.5 hours for samples with Kapton ${ }^{\circledR}$ tape). Upon inspection of the samples, the cause of the leakage was determined to be delamination of the tapes from the electrodes. In contrast, the samples packaged by laminated pallette paper revealed no signs of current leakage. The results imply that the latter method produces a satisfactory mositure barrier and can be reliably used for packaging sensors to be used in wet environments such as wounds. 


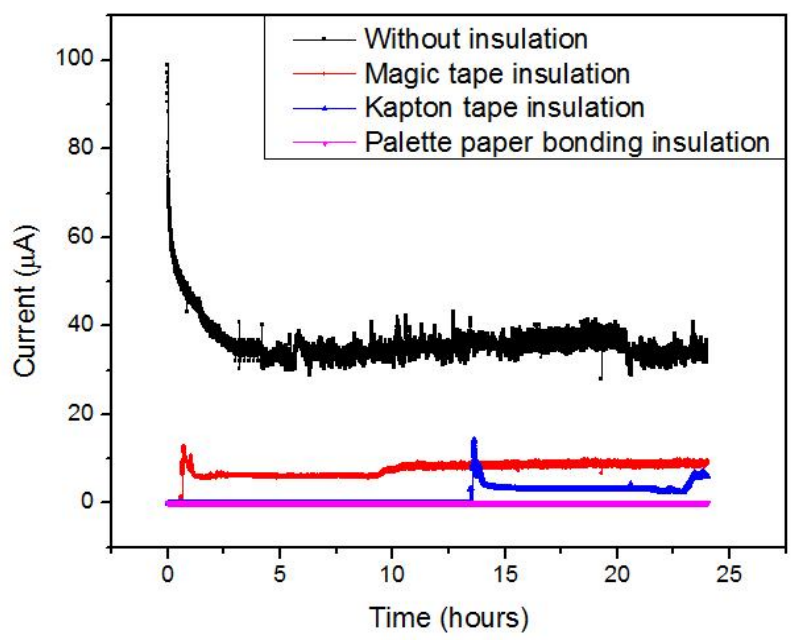

Figure 7: Electrical isolation properties of the palette paper bonding compared to Scotch ${ }^{\circledR}$ MagicTape ${ }^{T M}$ and Kapton ${ }^{\circledR}$ tapes. The current was measured across two electrodes with different isolations materials in a PBS solution with an applied voltage bias $(1 \mathrm{~V})$. The data reveal the superior performance of thermallylaminated palette paper.

\section{CONCLUSION}

An inexpensive, flexible array of $\mathrm{pH}$ sensors was fabricated on a paper substrate for use in wound monitoring. The developed fabrication process takes advantage of lowcost materials, laser machining, and self-aligned passivation with lamination technology. The sensor performance in different buffer solutions of $\mathrm{pH} 4$ to $\mathrm{pH} 10$ showed a linear potential $\left(r^{2}=0.9734\right)$ with and a sensitivity of $-49.5 \mathrm{mV} / \mathrm{pH}$. The obtained sensors features good flexibility, sensitivity and repeatability making it appropriate for integration with lowcost wound monitoring dressings.

\section{ACKNOWLEDGEMENTS}

The authors thank the staff of the Birck Nanotechnology Center for their assistance in this work. This work was funded by the National Science Foundation under the EFRI Program, grant $\# 1240443$.

\section{REFERENCES}

[1] L. A. Schneider, A. Korber, S. Grabbe, and J. Dissemond, "Influence of $\mathrm{pH}$ on wound-healing: a new perspective for wound-therapy?," Arch. Dermatol. Res., vol. 298, no. 9, pp. 413-20, Feb. 2007.

[2] S. Schreml, R. J. Meier, O. S. Wolfbeis, M. Landthaler, R.-M. Szeimies, and P. Babilas, "2D luminescence imaging of $\mathrm{pH}$ in vivo.," Proc. Natl. Acad. Sci. U. S. A., vol. 108, no. 6, pp. 2432-7, Feb. 2011.

[3] J. R. Sharpe, S. Booth, K. Jubin, N. R. Jordan, D. J. Lawrence-Watt, and B. S. Dheansa, "Progression of wound $\mathrm{pH}$ during the course of healing in burns.," J. Burn Care Res., vol. 34, no. 3, pp. e201-8, 2012.

[4] G. Urban, G. Jobst, F. Keplinger, E. Aschauer, O. Tilado, R. Fasching, and F. Kohl, "Miniaturized multi-enzyme biosensors integrated with $\mathrm{pH}$ sensors on flexible polymer carriers for in vivo applications," Biosens. Bioelectron., vol. 7, no. 10, pp. 733-739, Jan. 1992.
[5] O. Korostynska, K. Arshak, E. Gill, and A. Arshak, "Review on State-of-the-art in Polymer Based pH Sensors," no. 1, pp. 3027-3042, 2007.

[6] D. Lee and T. Cui, "Low-cost, transparent, and flexible single-walled carbon nanotube nanocomposite based ionsensitive field-effect transistors for $\mathrm{pH} /$ glucose sensing.,' Biosens. Bioelectron., vol. 25, no. 10, pp. 2259-64, Jun. 2010.

[7] V. Pachauri, K. Kern, and K. Balasubramanian, "sensitive metal-semiconductor field-effect transistor Field-effect-based chemical sensing using nanowire-nanoparticle hybrids: The ion-sensitive metal-semiconductor field-effect transistor," vol. 023501, 2013.

[8] C.-S. Lee, S. K. Kim, and M. Kim, "Ion-sensitive field-effect transistor for biological sensing.," Sensors (Basel)., vol. 9, no. 9, pp. 7111-31, Jan. 2009.

[9] W.-D. Huang, H. Cao, S. Deb, M. Chiao, and J. C. Chiao, "A flexible $\mathrm{pH}$ sensor based on the iridium oxide sensing film," Sensors Actuators A Phys., vol. 169, no. 1, pp. 1-11, Sep. 2011.

[10] M. Yuqing, C. Jianrong, and F. Keming, "New technology for the detection of pH.," J. Biochem. Biophys. Methods, vol. 63, no. 1, pp. 1-9, Apr. 2005.

[11] P. J. Kinlen, J. E. Heider, and D. E. Hubbard, "A solid-state $\mathrm{pH}$ sensor based on a Nafion-coated iridium oxide indicator electrode and a polymer-based silver chloride reference electrode," Sensors Actuators B Chem., vol. 22, no. 1, pp. 1325, Oct. 1994.

[12] S. Kakooei, M. C. Ismail, and B. Ari-wahjoedi, "Review Article An overview of pH Sensors Based on Iridium Oxide : Fabrication and Application," vol. 1, no. 1, pp. 62-72, 2013.

[13] O. Korostynska, K. Arshak, E. Gill, and A. Arshak, "Review Paper: Materials and Techniques for In Vivo $\mathrm{pH}$ Monitoring," vol. 8, no. 1, pp. 20-28, 2008.

[14] P. Kurzweil, Metal Oxides and Ion-Exchanging Surfaces as pH Sensors in Liquids: State-of-the-Art and Outlook., vol. 9, no. 6. 2009, pp. 4955-85.

[15] A. Richter, G. Paschew, S. Klatt, J. Lienig, K. Arndt, and H. P. Adler, "Review on Hydrogel-based pH Sensors and Microsensors," vol. c, pp. 561-581, 2008.

[16] V. Sridhar and K. Takahata, "A hydrogel-based passive wireless sensor using a flex-circuit inductive transducer," Sensors Actuators A Phys., vol. 155, no. 1, pp. 58-65, Oct. 2009.

[17] A. Nocke, A. Schröter, C. Cherif, and G. Gerlach, "Miniaturized textile-based multi-layer ph-sensor for wound monitoring applications," Autex Res. J., vol. 12, no. 1, pp. 20 22, Jan. 2012.

[18] P. Malkaj, E. Dalas, E. Vitoratos, and S. Sakkopoulos, "pH electrodes constructed from polyaniline/zeolite and polypyrrole/zeolite conductive blends," J. Appl. Polym. Sci., vol. 101, no. 3, pp. 1853-1856, Aug. 2006.

[19] B. Lakard, O. Segut, S. Lakard, G. Herlem, and T. Gharbi, "Potentiometric miniaturized $\mathrm{pH}$ sensors based on polypyrrole films," Sensors Actuators B Chem., vol. 122, no. 1, pp. 101108, Mar. 2007.

[20] K. Hamdani and K. L. Cheng, "Polyaniline pH Electrodes," Microchem. J., vol. 61, no. 3, pp. 198-217, Mar. 1999.

\section{CONTACT}

*B.Ziaie, tel:+1-765-404-0726; bziaie@ purdue.edu 